\title{
Correlations between inflammatory response and the increased lymphocyte percentage or CD19+ cell count in Kawasaki disease children undergoing intravenous immunoglobulin in different age intervals
}

Chun Zhang ( $\square$ chun_zhl@126.com )

Shanghai Jiao Tong University School of Medicine https://orcid.org/0000-0003-2771-2502

Xuan Zhang

Shanghai Jiao Tong University School of Medicine

Jia Shen

Shanghai Jiao Tong University School of Foreign Languages

Xiaotong Lu

Shanghai Jiao Tong University School of Medicine

Jian Zhang

Shanghai Jiao Tong University School of Medicine

Sun Chen

Shanghai Jiao Tong University School of Medicine

\section{Research article}

Keywords: Kawasaki disease (KD); inflammation; intravenous immunoglobulin (IVIg); neutrophil percentage; lymphocyte percentage; interleukin-10 (IL-10)

Posted Date: August 15th, 2019

DOl: https://doi.org/10.21203/rs.2.12948/v1

License: (c) (i) This work is licensed under a Creative Commons Attribution 4.0 International License.

Read Full License 


\section{Abstract}

Background: Intravenous immunoglobulin (IVIg) is used commonly in Kawasaki Disease (KD) but the inflammatory condition during this course remains unclear. We evaluated the inflammatory response before and after the performance of IVIg in KD children in different age intervals to explore precise markers of inflammation and to help individualized treatment of IVIg Methods: A retrospective survey involving $60 \mathrm{KD}$ children was performed. According to different age intervals, all patients were divided into 5 groups. Linear regression and correlation analysis were performed between the percentage of decreased neutrophil and that of increased lymphocyte in all groups and so did between the absolute cell count of CD19 + cells and the level of interleukin-10 (IL-10) in patients aged 4 and over. Results: During the acute phase of KD, after the treatment of IVIg all patients' reduced percentage of neutrophil correlated positively well with the increased percentage of lymphocyte. With age increased, the correlation coefficients valued higher which were 0.91 (group of less than 1 year), 0.87 (1 year), 0.91 (2 years), 0.97 (3 years), 0.99 (4 years and over) respectively with $p$ value less than 0.01 in all groups. Growing age obviously correlated positively with the value of correlation coefficient $(r=0.88, P<0.05)$. In the patients aged 4 and over, abnormal higher CD19 + cell absolute count before the administration of IVIg correlated positively with the scope of reduced IL-10 after the performance of $I V I g(r=0.71, P<0.05)$. Conclusions: During the acute phase of KD children with older age, the regulatory effect of IVIg on the immune response was better and the following recovery of immune function was more rapidly. In KD children aged 4 years and over the abnormal proliferating CD19 + B cell played dominant role in active humoral immune and may have the function of the IL-10 secretion as a compensatory response to balance the overactive humoral immune. Combined assessment of CD19 + B cell absolute count and the extent of IL10 reduction after IVIg may be a marker of therapeutic effect of IVIg on inflammatory immune response in older KD children.

\section{Background}

The leading causes of acquired heart diseases in pediatrics which are coronary arteries lesions (CALs) including dilations and aneurysms come from the serious complications of Kawasaki Disease (KD) [1]. $\mathrm{KD}$ is an acute, self-limiting systemic inflammation and commonly occurs as vasculitis especially in young children less than 5 years. During the acute period of this disease, intravenous immunoglobulin (IVIg) timely is the predominant therapeutic regimen with well-established efficacy [2]. However, the curative effect of IVIg can be evaluated only by the amelioration of clinic symptoms such as fever, rash, conjunctivitis and so on. The impact of IVIg on the change of KD children's laboratory data in inflammatory condition has not been fully delineated and the underlying mechanism has not been demonstrated clearly.

A child's immune system is a process of continuous development and mature from infancy to adult especially in one's early life. Children when growing are facing different antigen stimulations as infections, vaccinations, and so on from exterior. Both the evolution and the extreme genetic polymorphism selected by the environmental pressure develop a gradually working and mature immune 
response, which is different from the number, the repertoire and the acquired memory in immune cell according to the rise of children's age [3]. Therefore, it is understandable the inflammatory response may have great difference in KD children with different ages. It is necessary to delineate the changing trend of the major cell subset involved in the immune response so to understand clearly the basic pathogenic mechanism of therapy and find more accurate potential target to prevent the occurrence of complications from KD and identify the vulnerable host.

Numbers of evidence and data from clinical features support that bacterial infection is an important inducer of KD. The response of neutrophil play dominant role in the early and acute phase of KD manifested as the increase number of neutrophil in the peripheral blood, the infiltration on the necrotizing arteritis [4]. What's more, it has reported the neutrophil -to -lymphocyte ratio is useful to evaluate the inflammatory state in KD patients $[5,6]$. Thus, it is meaningful to investigate the distribution of these two blood cell subtypes before and after IVIg in KD children with different age.

Previous reports revealed the plasma level of interleukin-10 (IL-10) in KD patients during the acute phase are obviously higher than the levels of the patients in the convalescent phase and those of the control children [7]. Another study has found that elevated IL-10 levels during the acute phase of KD decreased immediately after IVIg administration, coincidentally with rapid improvement of inflammatory symptoms. It was detected the human IL-10 expressions from human gene and mRNA in both T- and B-cells [8]. However, this study did not demonstrate further the definite relationship between IL-10 and the T- or Bcells or the sequent effect on the immune response. Further study revealed that the IL-10 genetic polymorphisms have important effects on the CALs in acute KD patients and the ATA genotyping is associated with significantly increased risk of CALs [9]. It has found recently that IL-10 as an immuneregulatory cytokine has function of switch for lympho-proliferation in human $\mathrm{T}$ cell leukemia associating disease in addition to the suppressive effect on the Th1 response and inflammation [10]. It is an inquiry to us the role of IL-10 and its correlated relation to the subtype of lymphocyte during the incidence and therapy of acute phase of KD.

Focusing on the inflammatory reaction in KD children with different age before and after IVIg, the aim of the present investigation is to delineate the immune regulation in the change of lymphocyte subgroup and cytokine, explore precise markers of inflammation and find out the possible molecular mechanism underlying the effect of IVIg. Further, it is expecting to evaluate the therapeutic efficacy accurately from the point of inflammatory feature to help individualized treatment of IVIg.

\section{Methods}

\section{Setting of the study}

This retrospective study was conducted in Xinhua Hospital, which is not only a comprehensive hospital affiliated Shanghai Jiao Tong University School of medicine but also contains pediatric medical centre, one of four major pediatric hospitals where children affected by KD are treated in Shanghai, a city more than 20 million people living in. 


\section{Patients}

60 children diagnosed with KD [11] during April to December in 2018 in Xinhua Hospital affiliated to Shanghai Jiao Tong University School of Medicine were enrolled in this retrospective study. All patients

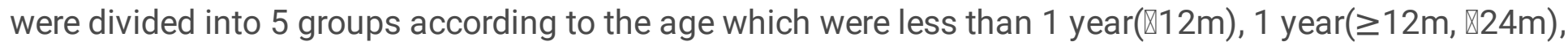

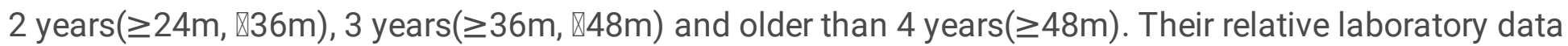
were collected and made a statistical analysis. Children with heart, lung, or kidney disease were excluded. The study was approved by the Ethics Committee in Xinhua Hospital Affiliated to Shanghai Jiao Tong University $\mathbb{}$ School of Medicine.

\section{Procedures and samples}

All the children received intravenous immune globulin (IVIg) treatment. Once diagnosed with KD, the child would receive IVlg therapy within $24 \mathrm{~h}$ with the single dose of $2 \mathrm{~g} / \mathrm{kg}$. Before the implement of IVlg and on the following third day of IVIg administrated all children were tested the white blood cell differential count by full automatic hematology analyzer and the inflammatory cytokines using solid-phase enzyme labeled chemiluminescent immunometric assay (Siemens Healthcare Ltd. US). Lymphocyte subsets were detected by flow cytometry (BD canto plus, US) before IVIG.

\section{Statistical analysis}

Statistical analysis performed by using the Graphpad Prism6.0 software and Excel 2010. According to different age group, the percentage of neutrophil before IVIg used to reduce that after IVIg and the scope of reduced neutrophil was obtained. In the same way, we got the increase percentage of lymphocyte using the data before and after IVIg. All data compared with the normal value established in different age intervals from newly published reference [12]. Following that, linear-regression analysis was performed between the two scopes in different age groups. Specially, in the group of children aged 4 years and over, the decrease scope of IL-10 before and after IVIg was calculated and then linear-regression analysis was performed between the decrease scope of IL-10 and the absolute count of CD19 positive cells before IVIg. A two-tailed $P \otimes 0.05$ was considered to be statistically significant.

\section{Results}

All KD cases included 26 boys and 34 girls. From young to old, 5 groups consisted of $6,14,16,5$ and 9 children respectively. Respect to the 5 different age interval groups, there are no obvious difference in the distribution of sex (Tab.1).

\section{All patients' mean neutrophil percentage reduced and the mean lymphocyte percentage increased after IVlg administrated in all different age groups}

In all different age groups, during the acute phase of KD all patients' neutrophil percentage increased abnormal highly compared to that of the value in the healthy children newly published. Inversely all KD children's lymphocyte percentage of was obviously lower than the value in the healthy children [12]. After 
the treatment of IVIg, all patients' neutrophil percentage decreased and lymphocyte percentage increased obviously as shown in Table 2.

\section{Neutrophil percentage reduction correlated positively with the increased lymphocytes percentage in different age groups}

The reduced neutrophil percentage and the increased percentage of lymphocytes after IVlg were calculated. As shown in Fig. 1a-e, the scope of reduced neutrophil percentage correlated positively well with the increased lymphocytes percentage in all five age groups. The correlation coefficients from young to old group were $0.91(\mathrm{n}=6, P=0.0116), 0.87(\mathrm{n}=14, P<0.01), 0.91(\mathrm{n}=16, P<0.01), 0.93(\mathrm{n}=5, P=$ $0.0065), 0.98(\mathrm{n}=9, P<0.01)$. All the $p$ value were less than 0.01 . The similar correlation were neither found between the percentage of neutrophil and the platelet count or the percentage of monocyte nor between the percentage of lymphocyte and the platelet count or the percentage of monocyte.

Along with children growing older and maturing, the median age in each group correlated closer with the coefficients significantly (Fig. $1 \mathrm{f}, \mathrm{r}=0.93, P=0.03$ ) suggesting the higher correlation occurred between the increased scope of lymphocyte and reduced extent of neutrophil after the treatment of IVIg. Better regulatory effect of IVIg on the re-balance of the white cell subset number will attained in peripheral blood with older KD children as shown in Tab.2.

\section{CD19 positive $\left(\mathrm{CD} 19^{+}\right)$B cell absolute count before the administration of IVIg correlated positively with the reduced extent of IL-10 in the patients aged 4 and over.}

The correlation between the reduced percentage of neutrophil and the increased percentage of lymphocyte showed that the older the children were, the closer the two changed scopes correlated suggesting in older children who has developed more maturing immune system, the administration of IVIg leads better and faster recovery of inflammation. In the followed investigation, we chose the eldest group to study further the relationship between the subset of lymphocyte and the inflammatory response in order to evaluate the therapeutic effect of IVIg and the inflammatory state in patients. After analysis, the relationship between $\mathrm{CD} 19^{+} \mathrm{B}$ cell absolute count and the expression of IL-10 attracted our attention. In the patients aged 4 and over $(n=9)$, the mean value of $C D 19^{+}$cell absolute count was 495.11 cells / $\mu \mathrm{L}$ and the standard deviation was 250.5 cells $/ \mu \mathrm{L}$ which were significantly higher than the normal standardized threshold established in our hospital which are 224.5 cells $/ \mu \mathrm{L}$ and 152.03 cells $/ \mu \mathrm{L}(\mathrm{n} \geq$ $200)$.

At the same time, in this group the mean expression of IL-10 was as high as $21.34 \mathrm{pg} / \mathrm{ml}$ before the performance of IVIg, which was obviously higher than the normal value of threshold which is less than 5 $\mathrm{pg} / \mathrm{ml}$. After received the IVIg, the patients' IL-10 reduced significantly and the mean expression of IL-10 was $5.3 \mathrm{pg} / \mathrm{ml}$. As shown in Fig.2. The amount of the $\mathrm{CD} 19^{+}$cell before the IVIg correlated positively well with the scope of decreased IL-10 after the administration of IVIg. The correlation coefficient was 0.71 and the $p$ value is less than 0.05 . No correlations were found between other inflammation cytokines 
including IL-1, TNF-a, IL-2 receptor, IL- 6 and other subset groups of lymphocyte as CD $4^{+}$cell, CD $8^{+}$cell or CD $16^{+} 56^{+}$cell.

\section{Discussion}

In the presented study, 60 cases of KD patients were involved in this retrospective analysis and divided into 5 groups according to the children's different age interval. There are no obvious differences in the sex distribution as to the incidence of KD in all groups, which has a discrepancy with other studies showing the disease more frequently occurs in boys $[13,14]$ but is consistent with the data recently reported in Australia [15]. A larger number of patients enrolled will have more convictive conclusion in this issue.

Neutrophil recruitment is an early event induced by the bacteria infection except for severe trauma, massive haemorrhage, malignancy and chemical poisoning $[16,17]$ and lymphocyte loss is usually looked as early biomarkers for systemic spread of severe infection [18]. Results revealed that in the acute phase of KD the abnormal increase of mean percentage of neutrophil and decreased percentage of the lymphocyte before IVIg constituted predominant changeable feature according to KD children's laboratory parameters. The concurrence of increased neutrophil, decreased lymphocyte and the obvious correlations between the two indexes indicate that the bacteria infection existing in the KD process most likely acts as an inducer and therefore, the viral infection can be excluded. Particularly, the extent of reduced percentage of neutrophil and that of increased percentage of lymphocyte after the administration of IVIg correlated positively in all age patients in this study indicating a feature of this disease in patients, which showed the possible infection was controlled and the immune function was regulated directing to the normal level by the IVIg. It also suggests the use of antibiotics during the acute phase of KD may contribute to the improvement in KD and have a synergistic effect with the IVIg. Even if the therapeutic course of antibiotics has finished or stopped, post antibiotic effect may still exist.

As it known, a child's immunologic maturity will not reach until adolescence. Not until 2 years old, adaptive and immune responses could not largely approach those of healthy adult levels and full immune competence will not truly reach until the teenage years [19]. Therefore, it is understandable that the coefficient of correlation ( $r$ ) shows higher correlation in the scope changed between the neutrophil and the lymphocyte as the children grow older. As the mean of age rises, the correlated coefficient becomes higher. These results also suggest better immune-modulatory effect of IVIg usually obtained on children older than 4 years including the rapid balance of cell count changing to normal between neutrophil and lymphocyte.

Therefore, in the following investigation, the relationship between special subset of lymphocyte and the cytokines was in focus to study further. It found that among the patients aged 4 and over old, before the treatment of IVIg, both the means of absolute $\mathrm{CD} 19^{+} \mathrm{B}$ cell count and the level of IL-10 were significantly higher than normal level. The more $\mathrm{CD}_{19}{ }^{+} \mathrm{B}$ cell absolute count was, the higher level of IL-10 was detected in the child. After the use of IVIg in the acute phase of KD, the inflammatory response regulated effectively manifesting as the anti-inflammatory cytokines IL-10 reduced obviously and the attenuated 
extent of IL-10 correlated positively well with the absolute count of increased CD19+ $\mathrm{B}$ cells assessed prior to the IVIg. CD19+ B cells is immunoglobulin production cells and play major role in the humoral immune response. The obviously higher count of $\mathrm{CD} 19^{+} \mathrm{B}$ cells before IVIg shows its activation and proliferation under the stimulation of antigen and indicates the predominant role of activated humoral immunity in the acute phase of KD children. The abnormal activation of humoral immunity response is also consistent to the speculation of the bacteria infection as an inducer of the KD. It has been reported previously that in mice CD19 enhances the $B$ cell receptor (BCR) induced signaling which is crucial for the activation and proliferation of $B$ cells and the following enhanced response of humoral immune $[20,21]$. This finding may be a new evidence as to our knowledge that proliferating $\mathrm{CD}^{1} 9^{+} \mathrm{B}$ cell plays dominant role in the active humoral immune in KD child older than 4 years.

What's more, the positive line correlation between the IL-10 and the absolute cell count of $\mathrm{CD} 19^{+}$suggests the possible ability of IL-10 secretion function of CD19+ B cells. IL-10 known as an anti-inflammatory cytokine was first described that it could be secreted in $T_{h} 2$ cells accompanied by other $T_{h}$ 2-type cytokines such as IL-4, IL-5 and so on in the classic immunology theory. Later, IL-10 was found to be secreted by other cell types including macrophages, DCs, mast cells even neutrophils [22]. Recently, the concept that B cell can inhibit immunity by the provision of IL-10 has been confirmed in mice. In mouse model, CD19-deficient mice displayed a reduced production of IL-10 by B cells and developed an exacerbated disease [23]. At the same time, another study has demonstrated that in mice reactive oxygen species could suppress humoral immune response through the reduction of CD19 expression and resultant BCR signaling [20]. In some patients treated with rituximab the depletion of B cell leaded to immunopathology [24]. It is also reported that $B$ cell from multiple sclerosis patients secreted markedly less IL-10 than B cells from healthy donors [25] suggesting the possible IL-10 secretion of B cells in some diseases of human. According to the presented result that the positive line correlation relationship between the $\mathrm{CD} 19^{+}$cell count and the reduced extent of IL-10, it is reasonable to speculate the proliferating $\mathrm{CD} 19^{+} \mathrm{B}$ cell may have the function of the IL-10 secretion as a compensatory response to balance the overactive humoral immune and regulatory effect on the immune response.

It is noticeable that after the treatment of IVIg, IL-10 reduced significantly suggesting the $\mathrm{CD} 19^{+}$cell absolute count may have a tendency of decrease wherever, the mean percentage of lymphocyte increased accompanied with the neutrophil decreased. As it is known the functions of immune globin are complex involving modulation of the expression and function of Fc receptors, interference with the activation of complement and the cytokine network, provision of antiidiotypic antibodies, and effector functions of T cells and B cells and so on [26]. Specially, IVIg could bind to the siglecs expressed on the surface of neutrophils and result in the cell death [27]. In addition, IVIg containing Fc has the ability to stimulate the expression of a population of natural regulatory T cells (nTreg) which could not be detected before IVlg treatment [28]. The phenomenon of decreased percentage of neutrophil and that of increased lymphocyte after IVIg is consistent to these conclusions. It could be reasoned that although the CD19+ $B$ cell count decreases, another major constitution of lymphocyte $T$ cells that also acting as an antigen presentation cell may be acted to increase after the use of IVIg and thus lead to the increase of 
percentage of lymphocyte as a whole in the KD pediatric patients. Therefore, by IVIg therapy the balance of the disordered immune responses tend to recover rapidly including the inhibition of hyper-humoral immune and the boost of cellular immune in KD children older than 4 years old whose immune system are approaching to maturation.

Taken together, $\mathrm{CD} 19^{+} \mathrm{B}$ cell absolute count may looked as an index to evaluate the seriousness of the humoral response in $\mathrm{KD}$ children. The combination of $\mathrm{CD} 19^{+} \mathrm{B}$ cell absolute count and the extent of reduced IL-10 post the IVIg could be considered as a marker to evaluate the sensitivity and therapeutic effect of IVIg on the inflammatory immune response in older KD children.

In this study, we put forward data in KD children aged 4 and over as we know for the first time that $\mathrm{CD} 19^{+} \mathrm{B}$ cell is stimulated to be active and proliferate directing to the enhanced function of humoral immune response, which has been verified in mice but have not clearly confirmed in human. Further, according to the results in this study combined the previous data reported, we proposes the speculation that $\mathrm{CD} 19^{+} \mathrm{B}$ cell in KD children may have the function of secreting the cytokine IL-10. However, more laboratory data need to be collected to confirm the conclusion.

From the strong positive correlation between the decreased neutrophil and the increased lymphocyte we come to the conclusion that the bacteria infection may be as an important inducer of KD and thus lead to the enhanced humoral immune response which is also consistent with the following change in peripheral blood white cell subgroup and the cytokine.

However, there are still some limitations in this retrospective analysis including the number of cases involved in are not fully enough and the data collected just from single one centre so that it could not completely reveal the overall perspective epidemiology of KD accurately.

Secondly, in the presented study the absolute cell count of subsets in lymphocyte especially the CD19+ cell should be traced to monitor dynamically after the IVIg. Therefore, the change tendency in the number of subset cell in lymphocyte could be analyzed more clear, the mechanism of IVIg and the inflammatory response in the special patients group could be demonstrated more definitely.

Another limitation is the infeasibility of function testify or cell deletion of CD19+ $B$ cells in patients so that the following response can be observed. However, it can tried in mice and some cell subset could be collected and cultured in vitro to verify the IL-10 secretion function of $\mathrm{CD} 19^{+} \mathrm{B}$ cell.

In the following study, more cases of KD children older than 4 years will be enrolled and more detailed examinations during the course of KD both before and after the IVIg therapy still have potential to refine in-depth the mechanism under the relationship between $\mathrm{CD}_{19}{ }^{+} \mathrm{B}$ cell count and the expression of IL-10.

\section{Conclusions}


IVIg shows ideal immune-modulatory effect on KD children in different age intervals manifesting as the attenuated scope of neutrophil percentage correlated positively with increased scope of lymphocyte percentage and suggesting the rapid recovery of balance obtained in the immune response. With children's age rises, the better effect of IVIg on KD children will be achieved. During the therapeutic period of IVIg, CD $19^{+} \mathrm{B}$ cells are stimulated to proliferate abnormally and plays dominant role in the activated humoral immune in KD child aged 4 years and over. The positive line correlation between the absolute cell count of $\mathrm{CD}_{19^{+}}$prior to IVIg and the decreased IL-10 post IVIg suggests the possible ability of IL-10 secretion function of $\mathrm{CD} 19^{+} \mathrm{B}$ cells in $\mathrm{KD}$ children aged 4 and over.

The combination of $\mathrm{CD} 19^{+} \mathrm{B}$ cell absolute count and the extent of reduced IL-10 post the IVIg could be considered as a marker to evaluate the sensitivity and therapeutic effect of IVIg in the inflammatory immune response in older KD children.

\section{Abbreviations}

BCR: B cell receptor; CD19: cluster of differentiation 19; CALs: coronary arteries lesions; IL-10: interleukin10; IVIg: intravenous immunoglobulin; KD: Kawasaki disease.

\section{Declarations}

\section{Funding}

In this study, Chun Zhang is supported by the Science Innovation Foundation of Shanghai Pharmaceutical Association (No. 2016-YY-01-06) and the Science Foundation of Medical School affiliated to Shanghai Jiao Tong University (No. 14XJ10033). Sun Chen is supported by Clinical Research Plan of SHDC (No. 16CR2021A).

\section{Availability of data and materials}

The data and materials of the study include personal medical records that are confidential and cannot be shared. All data supporting the statistical results reported in the article are kept in an electronic database by the corresponding author. They can be made available on reasonable request.

\section{Authors' contributions}

Chun Zhang and Sun Chen conceived of the idea for the project. Chun Zhang designed the study, performed the analysis of the data and wrote the paper. Xuan Zhang collected the data and formed the electronic database. Jia Shen admitted and treated patients. Xiaotong Lu and Jian Zhang were responsible for the coordination between departments. All authors contributed to the preparation of final manuscript. All authors read and approved of the final manuscript.

\section{Ethical approval and consent to participate}


All procedures performed in studies involving human participants were in accordance with the ethical standards of the institutional and/or national research committee of Xinhua Hospital affiliated to Shanghai Jiao Tong University School of Medicine and with the 1964 Helsinki declaration and its later amendments or comparable ethical standards.

Competing interests

The authors declare that they have no competing interests.

\section{Author details}

${ }^{[1]}$ Department of Pharmacy, Xinhua Hospital affiliated to Shanghai Jiao Tong University School of Medicine, Shanghai 200092, China. ${ }^{2}$ Department of Pediatric cardiology, Xinhua Hospital affiliated to Shanghai Jiao Tong University School of Medicine, Shanghai 200092, China.

\section{References}

References:

1. Dietz SM, van Stijn D, Burgner D, Levin M, Kuipers IM, Hutten BA, Kuijpers TW: Dissecting Kawasaki disease: a state-of-the-art review. EUR J PEDIATR 2017, 176(8):995-1009.

2. Burns JC, Franco A: The immunomodulatory effects of intravenous immunoglobulin therapy in Kawasaki disease. Expert Rev Clin Immuno/ 2015, 11(7):819-825.

3. Simon AK, Hollander GA, McMichael A: Evolution of the immune system in humans from infancy to old age. Proc Biol Sci 2015, 282(1821):20143085.

4. Newburger JW: Kawasaki disease: State of the art. CONGENIT HEART DIS 2017, 12(5):633-635.

5. Takeshita S, Kanai T, Kawamura Y, Yoshida Y, Nonoyama S: A comparison of the predictive validity of the combination of the neutrophil-to-lymphocyte ratio and platelet-to-lymphocyte ratio and other risk scoring systems for intravenous immunoglobulin (ivig)-resistance in Kawasaki disease. PLOS ONE 2017, 12(5):e176957.

6. Kawamura Y, Takeshita S, Kanai T, Yoshida Y, Nonoyama S: The Combined Usefulness of the Neutrophil-to-Lymphocyte and Platelet-to-Lymphocyte Ratios in Predicting Intravenous Immunoglobulin Resistance with Kawasaki Disease. J Pediatr 2016, 178:281-284.

7. Hirao J, Hibi S, Andoh T, Ichimura T: High levels of circulating interleukin-4 and interleukin-10 in Kawasaki disease. Int Arch Allergy Immunol 1997, 112(2):152-156.

8. Noh GW, Lee WG, Lee W, Lee K: Effects of intravenous immunoglobulin on plasma interleukin-10 levels in Kawasaki disease. IMMUNOL LETT1998, 62(1):19-24.

9. Weng KP, Hsieh KS, Hwang YT, Huang SH, Lai TJ, Yuh YS, Hou YY, Lin CC, Huang SC, Chang CK et al: IL-10 polymorphisms are associated with coronary artery lesions in acute stage of Kawasaki disease. CIRC J 2010, 74(5):983-989. 
10. Sawada L, Nagano Y, Hasegawa A, Kanai H, Nogami K, Ito S, Sato T, Yamano Y, Tanaka Y, Masuda T et al: IL-10-mediated signals act as a switch for lymphoproliferation in Human T-cell leukemia virus type-1 infection by activating the STAT3 and IRF4 pathways. PLOS PATHOG 2017, 13(9):e1006597.

11. McCrindle BW, Rowley AH, Newburger JW, Burns JC, Bolger AF, Gewitz M, Baker AL, Jackson MA, Takahashi M, Shah PB et al: Diagnosis, Treatment, and Long-Term Management of Kawasaki Disease: A Scientific Statement for Health Professionals From the American Heart Association. CIRCULATION2017, 135(17):e927-e999.

12. Zhang $X$, Ding $Y, Z$ Zhang $Y$, Xing J, Dai $Y$, Yuan E: Age- and sex-specific reference intervals for hematologic analytes in Chinese children. INT J LAB HEMATOL 2019, 41(3):331-337.

13. Uehara R, Belay ED: Epidemiology of Kawasaki disease in Asia, Europe, and the United States. $J$ EPIDEMIOL 2012, 22(2):79-85.

14. Salo E, Griffiths EP, Farstad T, Schiller B, Nakamura Y, Yashiro M, Uehara R, Best BM, Burns JC: Incidence of Kawasaki disease in northern European countries. PEDIATR INT 2012, 54(6):770-772.

15. Binder E, Griesmaier E, Giner T, Sailer-Hock M, Brunner J: Kawasaki disease in children and adolescents: clinical data of Kawasaki patients in a western region (Tyrol) of Austria from 20032012. Pediatr Rheumatol Online J 2014, 12(1):37.

16. de Oliveira S, Rosowski EE, Huttenlocher A: Neutrophil migration in infection and wound repair: going forward in reverse. NAT REV IMMUNOL 2016, 16(6):378-391.

17. Brinkmann V, Reichard U, Goosmann C, Fauler B, Uhlemann Y, Weiss DS, Weinrauch Y, Zychlinsky A: Neutrophil extracellular traps kill bacteria. SCIENCE 2004, 303(5663):1532-1535.

18. Loof TG, Sohail A, Bahgat MM, Tallam A, Arshad H, Akmatov MK, Pils MC, Heise U, Beineke A, Pessler F: Early Lymphocyte Loss and Increased Granulocyte/Lymphocyte Ratio Predict Systemic Spread of Streptococcus pyogenes in a Mouse Model of Acute Skin Infection. Front Cell Infect Microbio/ 2018, 8:101.

19. Randolph AG, McCulloh RJ: Pediatric sepsis: important considerations for diagnosing and managing severe infections in infants, children, and adolescents. VIRULENCE 2014, 5(1):179-189.

20. Ogura M, Inoue T, Yamaki J, Homma MK, Kurosaki T, Homma Y: Mitochondrial reactive oxygen species suppress humoral immune response through reduction of CD19 expression in B cells in mice. EUR J IMMUNOL 2017, 47(2):406-418.

21. Fujimoto M, Fujimoto Y, Poe JC, Jansen PJ, Lowell CA, DeFranco AL, Tedder TF: CD19 regulates Src family protein tyrosine kinase activation in $B$ lymphocytes through processive amplification. IMMUNITY 2000, 13(1):47-57.

22. Saraiva M, O'Garra A: The regulation of IL-10 production by immune cells. NAT REV IMMUNOL 2010, 10(3):170-181.

23. Matsushita T, Fujimoto M, Hasegawa M, Komura K, Takehara K, Tedder TF, Sato S: Inhibitory role of CD19 in the progression of experimental autoimmune encephalomyelitis by regulating cytokine response. AM J PATHOL 2006, 168(3):812-821. 
24. Hilgenberg E, Shen P, Dang VD, Ries S, Sakwa I, Fillatreau S: Interleukin-10-producing B cells and the regulation of immunity. Curr Top Microbiol Immunol 2014, 380:69-92.

25. Duddy M, Niino M, Adatia F, Hebert S, Freedman M, Atkins H, Kim HJ, Bar-Or A: Distinct effector cytokine profiles of memory and naive human $B$ cell subsets and implication in multiple sclerosis. $J$ IMMUNOL 2007, 178(10):6092-6099.

26. Kazatchkine MD, Kaveri SV: Immunomodulation of autoimmune and inflammatory diseases with intravenous immune globulin. N Engl J Med 2001, 345(10):747-755.

27. von Gunten S, Schaub A, Vogel M, Stadler BM, Miescher S, Simon HU: Immunologic and functional evidence for anti-Siglec-9 autoantibodies in intravenous immunoglobulin preparations. BLOOD 2006, 108(13):4255-4259.

28. Burns JC, Touma R, Song Y, Padilla RL, Tremoulet AH, Sidney J, Sette A, Franco A: Fine specificities of natural regulatory $\mathrm{T}$ cells after IVIG therapy in patients with Kawasaki disease. AUTOIMMUNITY 2015, 48(3):181-188.

\section{Tables}

Table 1 KD Children basic characteristics

\begin{tabular}{cccccc}
\hline$<1 \mathrm{y}$ & $1 \mathrm{y}$ & $2 \mathrm{y}$ & $3 \mathrm{y}$ & $\geq 4 \mathrm{y}$ \\
& $(<12 \mathrm{~m})$ & $(\geq 12 \mathrm{~m},<24 \mathrm{~m})$ & $(\geq 24 \mathrm{~m},<36 \mathrm{~m})$ & $(\geq 36 \mathrm{~m},<48 \mathrm{~m})$ & $(\geq 48 \mathrm{~m})$ \\
& $N=6$ & $N=14$ & $N=16$ & $N=5$ & $N=9$ \\
& $(100 \%)^{\mathrm{a}}$ & $(100 \%)^{\mathrm{a}}$ & $(100 \%)^{\mathrm{a}}$ & $(100 \%)^{\mathrm{a}}$ & $(100 \%)^{\mathrm{a}}$ \\
\hline Age (months) & 8.5 & 16.5 & 30 & 43 & 64 \\
& $(4.00-10.75)$ & $(15.25-18.75)$ & $(27.50-31.00)$ & $(42.00-43.00)$ & $(59.00-74.00)$ \\
\hline Sex, male & $3(50.0)$ & $9(64.3)$ & $8(50.0)$ & $2(40.0)$ & $4(44.4)$ \\
\hline
\end{tabular}

${ }^{a}$ Absolute number (percentage)

${ }^{b}$ Median (IQR) 
Table 2 Patients characteristics and the mean percentage neutrophil and lymphocyte before and after the treatment of IVIg in different age groups

\begin{tabular}{|c|c|c|c|c|c|c|c|c|c|c|}
\hline & $<1 \mathrm{y}$ & & $1 \mathrm{y}$ & & $2 y$ & & $3 y$ & & $\geq 4 \mathrm{y}$ & \\
\hline & $\mathrm{n}=6$ & & $\mathrm{n}=14$ & & $\mathrm{n}=16$ & & $\mathrm{n}=5$ & & $\mathrm{n}=9$ & \\
\hline & Before & After & Before & After & Before & After & Before & After & Before & After \\
\hline & IVIg & IVIg & IVIg & IVIg & IVIg & IVIg & IVIg & IVIg & IVIg & IVIg \\
\hline $\begin{array}{l}\text { Mean scope of neutrophil } \\
\qquad(\%)\end{array}$ & 58.45 & 27.53 & 57.92 & 28.4 & 68.26 & 39.18 & 62.36 & 29.94 & 80.22 & 45.64 \\
\hline $\begin{array}{l}\text { Mean scope of } \\
\text { lymphocyte (\%) }\end{array}$ & 28.17 & 53.42 & 31.34 & 53.47 & 22.79 & 45.22 & 26.58 & 52.56 & 13.02 & 40.94 \\
\hline $\begin{array}{c}\text { Mean scope of decrease } \\
\text { neutrophil (\%) }\end{array}$ & 30.92 & & 29.52 & & 29.09 & & 32.42 & & 34.58 & \\
\hline $\begin{array}{c}\text { Mean scope of increased } \\
\text { lymphocyte (\%) }\end{array}$ & 25.25 & & 22.14 & & 22.43 & & 25.98 & & 27.92 & \\
\hline
\end{tabular}

\section{Figures}



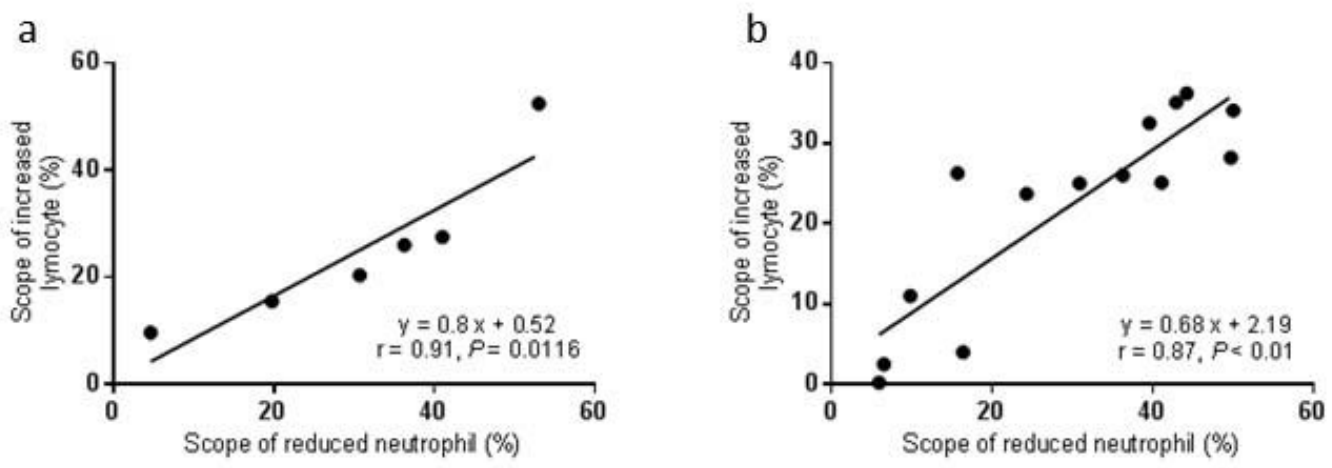

C

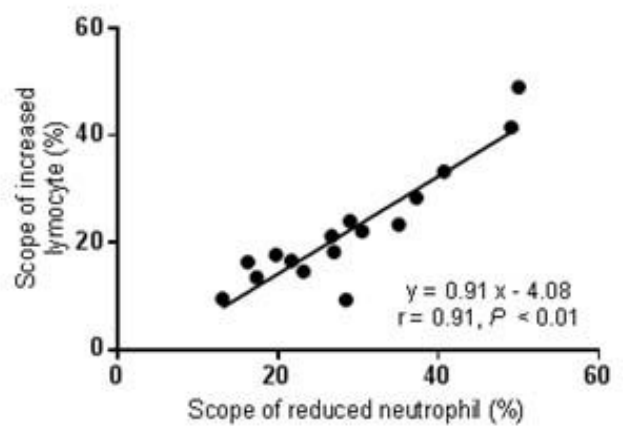

d
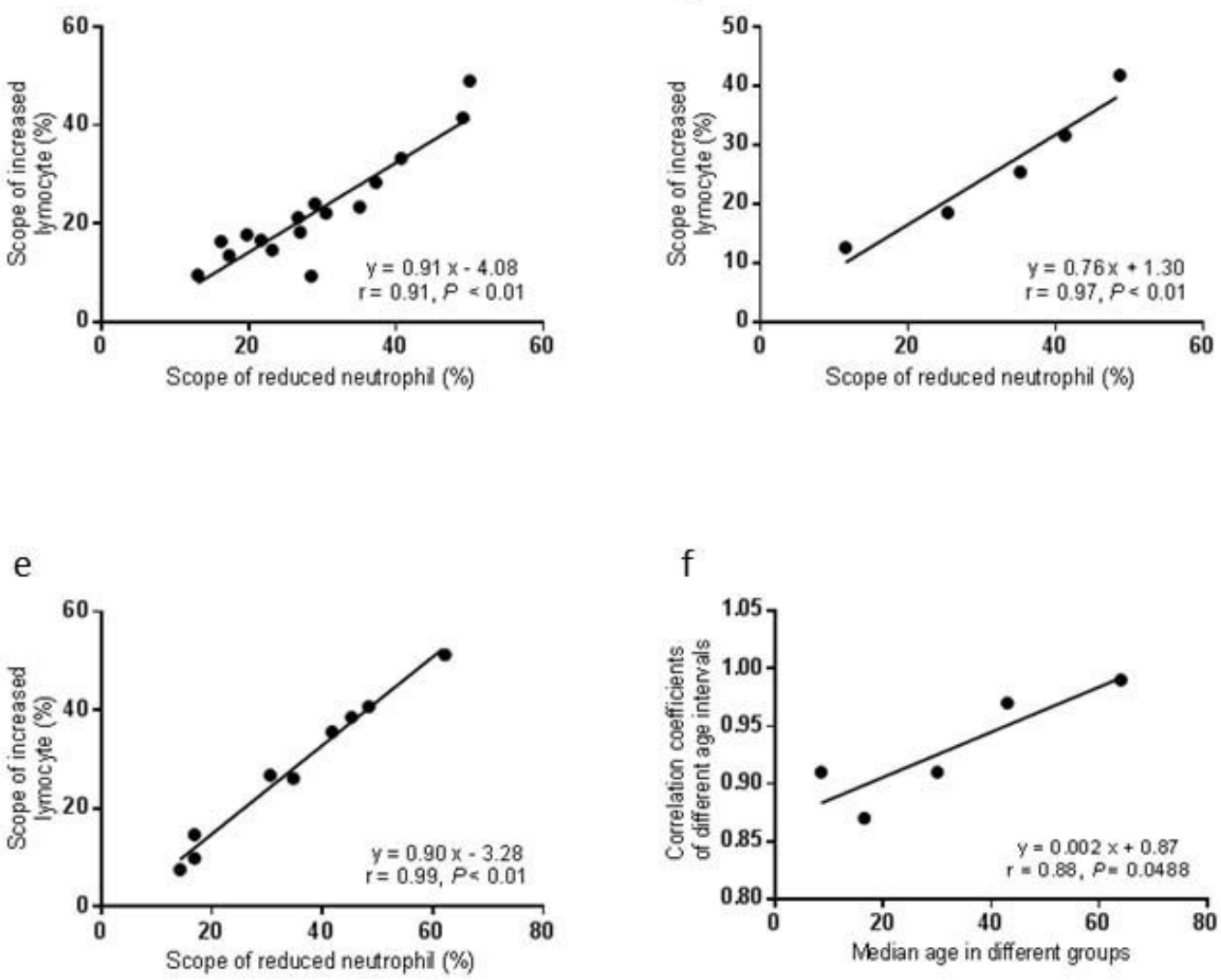

\section{Figure 1}

Correlation between percentage decrease of neutrophils and percentage increase of lymphocytes after IVIg in different age groups. The Spearman correlation coefficients ( $r$ ) from young to old group were 0.91 $(n=6), 0.87(n=14), 0.91(n=16), 0.97(n=5), 0.99(n=9)$, respectively. a. Less than 1 year; b. 1 year; c. 2 years. d. 3 years. e. 4 years and older than 4 years. F. Positive correlation between the mean median age and the correlation coefficients in different group. The $r$ is 0.88 and the $p$ value is 0.0488 . 


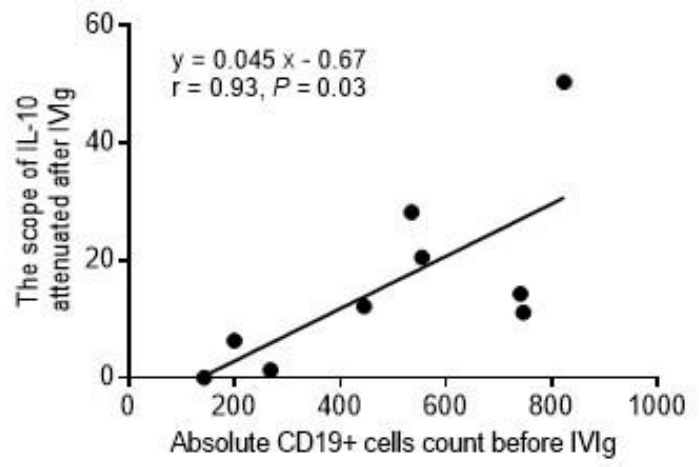

Figure 2

Positive correlation between the absolute CD19+ cell counts before IVIg and decrease of interleukin 10 (IL-10) after IVIg. The $r$ is 0.93 and the $p$ value is 0.03 in KD children aged 4 years and over. 